This is a postprint version of the following published document:

Sánchez-Arriaga, G.; Bombardelli, C. and Chen, X. Impact of nonideal effects on bare electrodynamic tether performance, in: Journal of Propulsion and Power, Vol. 31, No. 3 (2015), pp. 951-955.

DOI: https://doi.org/10.2514/1.B35393

(C) 2014 by the American Institute of Aeronautics and Astronautics, Inc. All rights reserved 


\title{
Impact of Nonideal Effects on Bare Electrodynamic Tether Performance
}

\author{
Gonzalo Sánchez-Arriaga,*- Claudio Bombardelli, $₫$ and Xin Chen ${ }^{ \pm}$ \\ Universidad Politécnica de Madrid, 28040 Madrid, Spain
}

DOI: $10.2514 / 1 . B 35393$

\begin{abstract}
The paper deals with three nonideal effects, often neglected in the literature, which affect the current and potential profiles along a bare electrodynamic tether. The first appears when the size of the tether cross-section width is large enough to have potential barriers for the probe radius in the radial effective potential energy of the plasma electrons. The tether is not able to capture the orbital-motion-limited current law and is said to operate beyond the orbitalmotion-limited regime. It is shown that this effect can be accurately modeled by just scaling the tether characteristic length according to a dimensionless factor depending on tether and plasma properties. The high-bias approximation in the orbital-motion-limited current collection law, normally assumed in past work, is discussed. The third effect becomes relevant when the cathodic plasma contactor potential drop is nonnegligible compared with the product of the tether length and the motional electricfield. Numerical simulations performed with a newly developed tether flight simulator across a wide range of mission scenarios and design parameters shows that the first effect can be safely ignored for preliminary tether mission design. The cathodic plasma contactor potential drop, however, increases the deorbit time of kilometer-length tethers in a nonnegligible way. In the extreme case in which the tether is less than a few hundred meters long, such an effect would strongly deteriorate its deorbit performance.
\end{abstract}

\begin{tabular}{lll} 
& \multicolumn{1}{c}{ Nomenclature } \\
$A$ & $=$ tether cross section \\
$B$ & $=$ geomagnetic field \\
$E_{m}$ & $=$ motional electric field \\
$e$ & $=$ electron charge \\
$h$ & $=$ tether thickness \\
$I$ & $=$ tether current \\
$I_{*}$ & $=$ tether short-circuit current \\
$L$ & $=$ tether length \\
$L^{*}$ & $=$ characteristic tether length \\
$m_{e, i}$ & $=$ electron (ion) mass \\
$N_{0}$ & $=$ unperturbed plasma density \\
$p$ & $=$ tether perimeter \\
$R$ & $=$ tether radius \\
$T_{e, i}$ & $=$ electron (ion) temperature \\
$u_{t}$ & $=$ unit vector from cathodic to anodic end \\
$v_{s}$ & $=$ tether-to-plasma relative velocity \\
$w$ & $=$ tether width \\
$\Delta V_{\mathrm{HC}}$ & $=$ hollow-cathode potential drop \\
$\sigma$ & $=$ tether conductivity \\
$\Phi$ & $=$ tether-to-plasma potential \\
$\Phi_{p}$ & $=$ plasma potential \\
$\Phi_{t}$ & $=$ tether potential
\end{tabular}

\section{Introduction}

B AREelectrodynamic tethers (EDTs), firstintroduced in 1991 by Sanmartin et al. [1], are an attractive device for deorbiting satellites and the upper stages of rockets [2]. The system consists of a few kilometers of an aluminum wire or tape left bare of insulation and a plasma contactor at its cathodic end. The good electric contact with

Received 25 March 2014; revision received 24 September 2014; accepted for publication 14 October 2014; published online 20 January 2015. Copyright 2014 by the American Institute of Aeronautics and Astronautics, Inc. All rights reserved. Copies of this paper may be made for personal or internal use, on condition that the copier pay the $\$ 10.00$ per-copy fee to the CopyrightClearance Center, Inc., 222 Rosewood Drive, Danvers, MA 01923; include the code 1533-3876/15 and $\$ 10.00$ in correspondence with the CCC.

*Assistant Professor, Departamento de Física Aplicada, Escuela Técnica Superior de Ingeniería Aeronáutica y del Espacio.

'Research Fellow, Departamento de Física Aplicada, Escuela Técnica Superior de Ingeniería Aeronáutica y del Espacio.

${ }^{\mp} \mathrm{Ph} . \mathrm{D}$. Candidate, Departamento de Física Aplicada, Escuela Técnica Superior de Ingeniería Aeronáutica y del Espacio. the surrounding plasma provided by such a configuration allows the tether to achieve a steady current, which is driven by the motional electric field appearing in the tether reference frame due to the tetherto-plasma relative velocity and the ambient magnetic field. The interaction of this current with the ambient magnetic field yields a drag force and the deorbiting of the satellite. Since the system is typically very light, propellantless, and does not require power supplies, EDTs are advantageous as compared to a thrust-based deorbiting method [3]. The performance of bare EDTs in low Earth orbit has been investigated by several authors in the past decade [1,38]. In particular, a relatively simple analytical model has been recently proposed to study the deorbiting time of tapelike, bare EDTs in inclined circular orbits [9].

Until recently, the main design criterion of a bare EDT was aimed at maximizing the ratio between the deorbiting force and the total tether mass, leading to thin and long tethers with tapelike cross sections of limited width (typically less than $2 \mathrm{~cm}$ ). The latestresults, however, have pointed out the need to minimize not just the tether mass but also the risk for the tether to be severed by small debris. This last issue rules out round tethers because their sever probability is about 1.5 orders of magnitude higher than a tape tether of equal mass and length [10]. Depending on the characteristic of the orbit (inclination and altitude) and the mass of the satellite, the tether can have an optimal width well above $2 \mathrm{~cm}$ and an optimal length down to $3-5 \mathrm{~km}$. For such a wide tether, the classical current profile analysis should be revisited because the tether would not collect in the orbitalmotion-limited (OML) regime. In addition, the influence of the nonzero potential drop of the cathodic plasma contactor, typically larger than $20 \mathrm{~V}$, must also be considered, as it may be no longer negligible when compared to the total potential difference along the tether, particularly for high inclination orbits and tethers as short as $3 \mathrm{~km}$ or less.

The Langmuir probe theory provides the OML current law $I_{\mathrm{OML}}$. which applies (within acertain parameter range) to an infinitely long cylinder, at rest, in unmagnetized, collisionless, Maxwellian plasma [11]. It is an upper-limit current law that holds for a probe radius $R$ below a maximum radius $R_{\max }$. If $R>R_{\max }$, then barriers appear at the probe in the effective potential energy of the plasma electrons and the collected current $I$ is below $I_{\mathrm{OML}}$. Both $R_{\max }[12]$ and the ratio $I / I_{\mathrm{OML}}$ for $R>R_{\max }$ [13] were computed using asymptotic theory in the limit of high bias; i.e., for a tether-to-plasma bias $\Phi$ and plasma temperature $T_{e} \sim T_{i}$ satisfying the condition $e \Phi / k T_{c, i} \gg 1$. These theories can be applied to tape tethers by just using the equivalent radius $R_{\text {eq }} \approx$ width $/ 4$ [12]. In past works, the high-bias approximation also was assumed to compute the tether current and 
potential profiles. The effect of a plasma velocity could play a role for a tether orbiting in the $F$ layer, where $\mathrm{O}^{+}$is the dominant ion species and the ion (ram) energy is large as compared with the thermal energy. This effect was analyzed by looking for self-consistent solutions of the stationary Vlasov-Poisson system [14]. However, stationary theory falls in a paradox [15]. Recent numerical simulations of the nonstationary Vlasov-Poisson system show that particle trapping happens at the tether front, thus explaining the paradox [16].

In Sec. II, we revisit $[1,3,4]$ and compute the current profile along the tether in operational conditions beyond the OML regime. Both the high-bias approximation and the exact current law are considered. A numerical parametric survey is carried out, and the importance of the different dimensionless parameters is highlighted. In Sec. III, we use the novel tether flight simulator software BETsMA to study the impact of the beyond-OMLeffect and the potential drop of the hollow cathode on tether performance. The results are discussed in Sec. IV.

\section{Current and Voltage Profiles}

We consider a rigid and fully bare tether of length $L$, cross-section area $A$, and perimeter $p$ made of a conductive material of uniform density $\rho$ and conductivity $\sigma$ (see Fig. 1). Due to the tether-to-plasma relative velocity $\boldsymbol{v}_{s}$ and the ambient magnetic field $\boldsymbol{B}$, an induced electric field along the tether $E_{m}=u_{t} \cdot\left(v_{s} \times B\right)$ appears in the tether frame. Here, $u_{t}$ is defined as the tangent unit vector along the tether pointing in the direction of the electric current. The spatial variation along the tether direction $x$ of the faraway plasma potential $\Phi_{p}$ is given by the motional electric field $E_{m}$ projected along the tether, whereas that of the tether potential $\Phi_{t}$ is due to ohmic losses. The potential bias $\Phi=\Phi_{t}-\Phi_{p}$ is then given by $[\underline{1,17]}$

$$
\frac{\mathrm{d} \Phi}{\mathrm{d} x}=\frac{I(x)}{A \sigma}-E_{m}
$$

where $I(x)$ is the current along the tether, and $0<x<L$.

For a bare tether, electrons are collected along the anodic length $0<x<L_{A B}$, where $\Phi>0$. They are ejected by some plasma contactor (e.g., a hollow cathode or a thermionic emitter device), at the cost of a potential drop $\Delta V_{\mathrm{HC}}$ at the cathodic end of the tether. An alternative to the Hollow-Cathode is a bare-tether with cathodic contact through thermionic emission by low-work-function materials [18]. The length $L_{A B}$, which is determined by the condition $\left.\overline{\Phi(x}=L_{A B}\right)=0$, depends on the plasma conditions and must be computed as part of the solution to the problem. For $0<x<L_{A B}$, two-dimensional probe theory provides the following law for the electron current collected by a probe with potential bias $\Phi$ [19]:

$$
\begin{aligned}
& \frac{\mathrm{d} I(x)}{\mathrm{d} x}=e N_{0} \frac{p}{\pi} \sqrt{\frac{2 e}{m_{e}}} \times G\left(\frac{e \Phi}{k T_{i}}, \frac{T_{e}}{T_{i}}, \frac{R}{\lambda_{D e}}\right) \\
& \times\left[\sqrt{\Phi}+\frac{1}{2} \sqrt{\frac{\pi k T_{e}}{e}} \exp \left(\frac{e \Phi}{k T_{e}}\right) \operatorname{erfc}\left(\sqrt{\frac{e \Phi}{k T_{e}}}\right)\right]
\end{aligned}
$$

where $T_{c}$ and $\lambda_{D e}\left(T_{i}\right.$ and $\left.\lambda_{D i}\right)$ are the electron (ion) temperature and Debye length, respectively. The terms inside the bracket correspond to the OML current collection law. The factor proportional to the complementary error function (erfe) is very small for high bias $\left(e \Phi / k T_{e} \gg 0\right)$, and it has been neglected in previous work.

The dimensionless function $G$ takes into account the appearance of potential barriers, which deviate the current collection from the OML regime. For given values of $T_{c} / T_{i}$ and $e \Phi / k T_{i}$, there are no potential barriers if the probe radius $R(R=w / 4$ for a tape of width $w)$ is below a maximum radius $R_{\max }$. For high bias $e \Phi / k T_{i} \gg 1, R_{\max }$ and the function $G$ were computed in [12] and [13] using asymptotic theory.

For convenience, we introduce the following characteristic magnitudes and dimensionless parameters $[3,17,19,20]$ :

$$
I_{*} \equiv E_{m} \sigma A, \quad \Phi_{*} \equiv E_{m} L_{*}, \quad \beta \equiv \frac{e E_{m} L_{*}}{k T_{i}}
$$
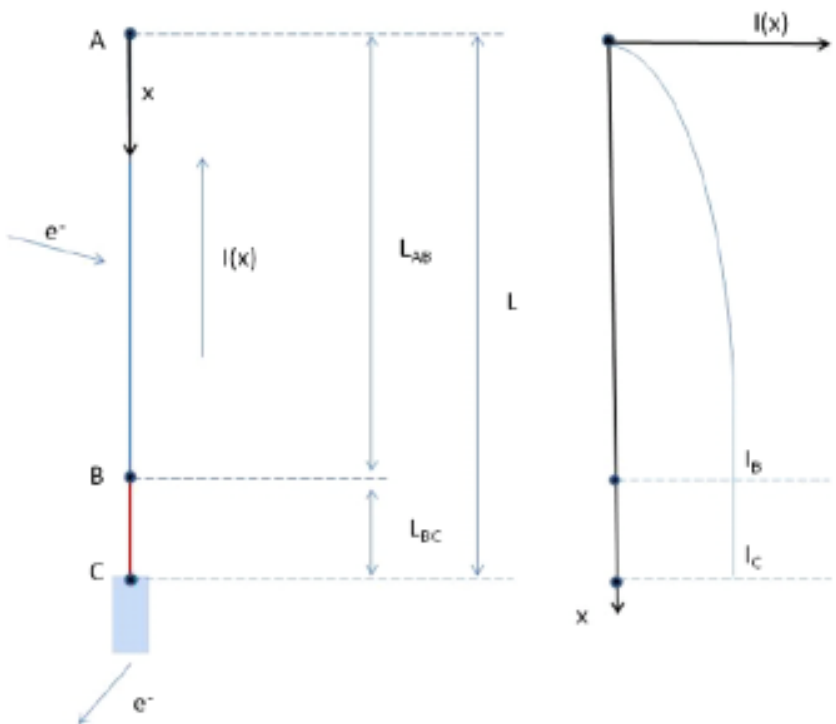

Fig. 1 Bare electrodynamic tether configuration.

$$
L_{*} \equiv\left(\frac{9 \pi^{2}}{128} \times \frac{1}{G^{2}\left(\beta, T_{e} / T_{i}, R / \lambda_{D e}\right)} \times \frac{m_{e} \sigma^{2}}{e^{3}} \times \frac{E_{m} h^{2}}{N_{0}^{2}}\right)^{1 / 3}
$$

where $h \equiv 2 A / p$. As compared with previous work $[1,3]$, the characteristic length $L_{*}$ differs by the factor $G^{2 / 3}$. Since $\beta$ is proportional to $L_{*}$, and it is one of the arguments of function $G$, Eq. (4) is an implicit equation for $L_{*}$. For a midinclination orbit of a height equal to $1000 \mathrm{~km}$, typical ambient values are $B \sim 0.3 G$, $N_{0} \sim 10^{11} \mathrm{~m}^{-3}, k T_{i} \sim k T_{e} \sim 0.2 \mathrm{eV}$, and $E_{m} \sim 100 \mathrm{~V} / \mathrm{km}$. A tether with $h \sim 0.1 \mathrm{~mm}$ would have $L_{*} \sim 2.7 \mathrm{~km}$ and $\beta \sim 1350$.

Using the dimensionless variables

$$
i \equiv \frac{I}{I_{*}}, \quad \phi \equiv \frac{\Phi}{\Phi_{*}}, \quad \xi \equiv \frac{x}{L_{*}}
$$

Eqs. (1) and (2) become

$$
\frac{\mathrm{d} \phi}{\mathrm{d} \xi}=i-1
$$

$$
\frac{\mathrm{d} i}{\mathrm{~d} \xi}=\frac{3}{4} \times \eta(\phi) \times\left[\sqrt{\phi}+\frac{1}{2} \sqrt{\frac{\pi}{\beta}} \exp (\beta \phi) \operatorname{erfc}(\sqrt{\beta \phi})\right]
$$

with

$$
\eta(\phi) \equiv G\left(\beta \phi, \frac{T_{e}}{T_{i}}, \frac{R}{\lambda_{D e}}\right) / G\left(\beta, \frac{T_{e}}{T_{i}}, \frac{R}{\lambda_{D e}}\right)
$$

As shown in [3], ion collection in the cathodic segment $\Delta I_{\mathrm{BC}}$ satisfies $\Delta I_{\mathrm{BC}} / I_{B} \sim \sqrt{\left(\left|\Phi_{C}\right| / \Phi_{A}\right)\left(m_{e} / m_{i}\right)}$. The ion current collection in this segment $\left(\xi_{B} \equiv L_{A B} / L_{*}<\xi<L / L_{*} \equiv \xi_{L}\right)$ can be safely neglected for the deorbiting mode $\left(\left|\Phi_{C}\right| / \Phi_{A}\right.$ and $m_{c} / m_{i}$ are small, typically), and we write $I(x) \sim I\left(L_{A B}\right) \sim I(L)$. The integration of Eq. (6a) in this region yields

$$
\phi_{C}=\left(1-i_{B}\right)\left(\xi_{L}-\xi_{B}\right)
$$

where we introduced the (positive) normalized potential drop $\phi_{C} \equiv\left|\Delta V_{\mathrm{HC}}\right| / E_{m} L_{*}$.

The dependence of the function $G$ on the tether normalized bias $e \Phi / k T_{i}$ is very weak. For instance, changing $e \Phi / k T_{i}$ from 300 to 3000 only produces a few percent of variation in $G$ [13]. This weak dependence is illustrated in Fig. 2 , which shows the function $\eta(\phi)$ for 


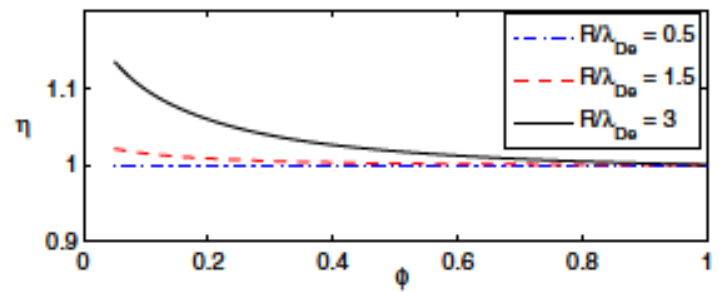

a)

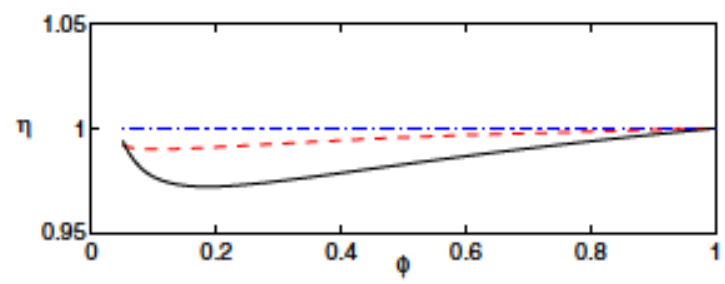

b)

Fig, 2 Function $\eta(\phi)$ with $T_{c} / T_{i}=1$. a) $\beta=100$, and b) $\beta=1000$.

$T_{c} / T_{i}=1$ and three different values of the ratio $R / \lambda_{D e}$. Function $G$ has been computed following the procedure described in [13]. Figures $2 \mathrm{a}$ and $2 \mathrm{~b}$ correspond to $\beta=100$ and $\beta=1 \overline{000}$, respectively. Clearly, $\eta$ is very close to one unless $\phi$ is very small: a range not considered in Fig. 2 because the high-bias assumption used in the asymptotic analysis in [13] breaks down. This range, however, can be safely ignored in the analysis because the right-hand side of Eq. (2) is of the order of the thermal current per unit length; $i_{\text {th }}=e N_{0} p \sqrt{k T_{c} / 2 \pi m_{e}}$.

The approximation $\eta(\phi) \approx 1$ simplifies the analysis considerably. The effect of the current collection drop due to a possible operation beyond the OML regime just appears in the definition of $L$, due to the factor $G^{-2 / 3}$. Since the current collected by each transverse section of the tether is a factor $G^{2 / 3}$ smaller due to the appearance of potential barriers, the characteristic length $L_{*}$ must be larger. Under the hypothesis $\eta \approx 1$, the mathematical problem of the beyond-OML tether operation [Eqs. (6a) and (6b)] is exactly equal to the OML one. We remark that parameters $\bar{T}_{c} / T_{i}, R / \lambda_{D i}$, and $e E_{m} L_{*} / k T_{c}$, involving tether and environmental properties, just appear in $L_{*}$; and they are decoupled from the determination of the normalized potential and current profiles.

If one assumes that $\eta \approx 1$, then the integration of Eqs. (6a) and (6b) in the anodic segment is straightforward. We first eliminate $\xi$ by using Eqs. (6a) and (6b). Imposing the boundary conditions $i(0)=0$ and $\phi(0)=\phi_{A}$, and $i\left(\xi_{B}\right)=i_{B}$ and $\phi\left(\xi_{B}\right)=0$, yields

$$
\begin{gathered}
1-\phi_{A}^{3 / 2}+g(0)-g\left(\phi_{A}\right)=\left(1-i_{B}\right)^{2} \\
\xi_{B}=\int_{0}^{\phi_{A}} \frac{\mathrm{d} \phi}{\sqrt{1-\left(\phi_{A}^{3 / 2}-\phi^{3 / 2}\right)+g(\phi)-g\left(\phi_{A}\right)}}
\end{gathered}
$$

where

$$
g(\phi) \equiv \frac{3}{4} \sqrt{\frac{\pi}{\beta^{3}}}\left[\exp (\beta \phi) \operatorname{erfc}(\sqrt{\beta \phi})+2 \sqrt{\frac{\beta \phi}{\pi}}\right]
$$

Equations (7), (8a), and (8b) give the unknowns $\phi_{A}, \xi_{B}$, and $i_{B}$. Once these variables are known, the integration of system 6 with condition $i=0$ and $\phi=\phi_{A}$ at $\xi=0$ gives the potential and current profiles $\phi(x)$ and $i(x)$. The normalized averaged current along the tether is

$$
i_{a v}=\frac{1}{L I_{*}} \int_{0}^{L} I(x) \mathrm{d} x=1-\frac{\phi_{A}+\phi_{C}}{\xi_{L}}
$$
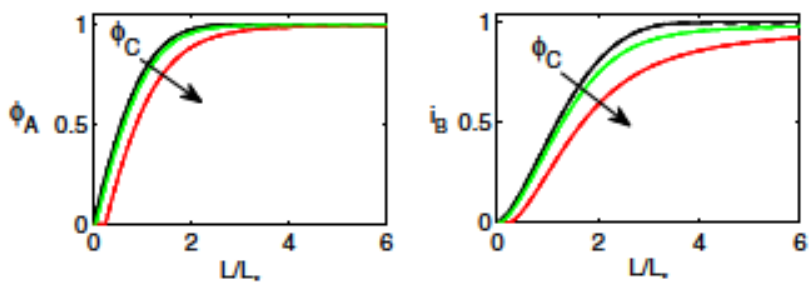

a)

b)
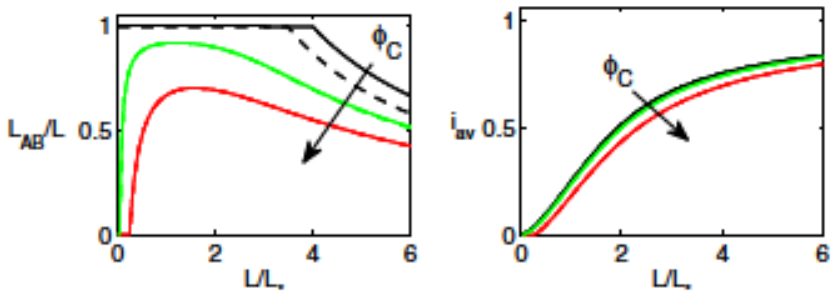

c)

d)

Fig, 3 The effect of the high bias hypothesis on tether performance.

Since the beyond-OMLeffect affects the definition of $L_{*}$, the novelty of Eqs. (7), (8a), and (8b) is the incorporation of the exact OML law. The solutions of this model are shown in Fig. 3. Solid lines correspond to the solutions of Eqs. (7), (8a), and (8b), ignoring the term proportional to the complementary error function (high-bias approximation); and dashed lines are their exact solutions for $\beta=1000$. Figures $3 \mathrm{a}-3 \mathrm{~d}$ show the characteristic values $\Phi_{A}, i_{B}$, $L_{A B} / L$, and $i_{a v}$ versus $L / L$, for $\phi_{c}=0,0.05$, and 0.25 . In Figs. $3 \mathrm{a}-$ $3 \mathrm{~d}$, solid and dashed lines practically overlap, indicating the goodness of the high-bias approximation for typical tether deorbiting missions. The most important discrepancy (of the order of $10 \%$ ) happens in $L_{A B} / L$ for the vanishing hollow-cathode potential drop and the so-called long tether regime. Numerical calculations reveal that the gap vanishes as $\beta \rightarrow \infty$ (not shown). We remark that such a difference has almost no impact on $i_{\mathrm{av}}$ [see Fig. 3d], which is the main quantity determining the performance of the tether as a deorbit device.

\section{Impact on Deorbit Performance}

The beyond-OML current collection model has been implemented in BETsMA, which is a new tether flight simulator developed under the FP7 project BETs [21]. This software, which includes a module to simulate the tether dynamics and a tool to determine the optimal tether geometry for a given mission, can be used to study the tether performance in a broad range of conditions. It includes DROMO [2224], a robust and efficient orbital propagator based on a new regularized orbital dynamics formulation.

A relevant scenario for space debris removal involves objects with masses of the order of 1 ton orbiting at an altitude between 800 and $1000 \mathrm{~km}$ [25]. Here, we consider a deorbit mission of a $1000 \mathrm{~kg}$ satel lite from 800 to $500 \mathrm{~km}$ of altitude with atether of length $3 \mathrm{~km}$, a width of $4 \mathrm{~cm}$, and thickness of $50 \mu \mathrm{m}$. The orbit of the satellite, initially circular, is perturbed by the Lorentz drag on the tether, which is assumed perfectly aligned with the local vertical. For simplicity, we assumed that the tether is equipped with two hollow cathodes at either end, i.e., the Lorentz force acts along the full orbit (even at high inclination, where a fraction of time the motional electric field would reverse its direction). The geomagnetic field and electron plasma density are computed with the International Geomagnetic Reference Field and the 2012 International Reference Ionosphere models, respectively. The starting date is set to 1 January 2005, which corresponds to a solar minimum. This set of hypotheses makes BET sMA a suitable software for preliminary mission design. However, more complex (and computationally expensive) tether simulations, including flexibility and lateral dynamics, would be necessary for detailed analysis. In general, optimal tether design 


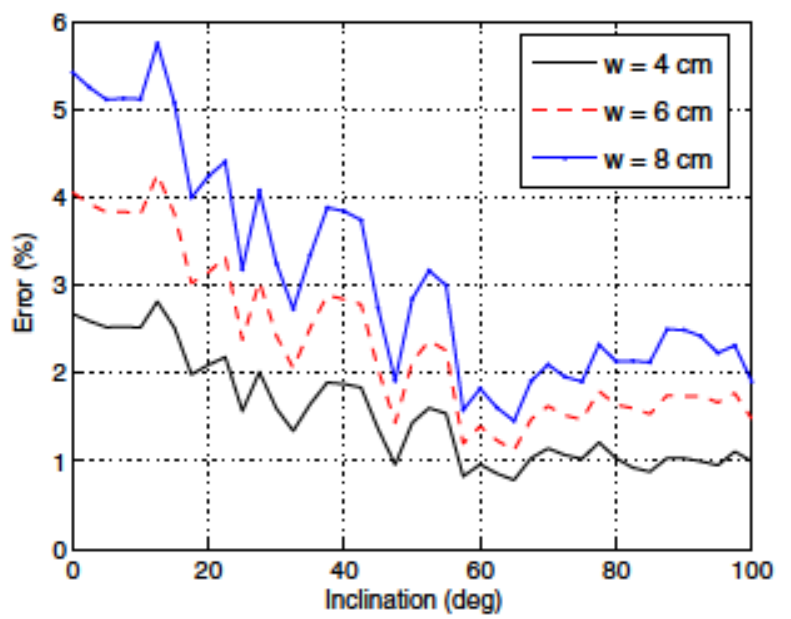

Fig 4 Deorbit time error if beyond-OML effect is ignored.

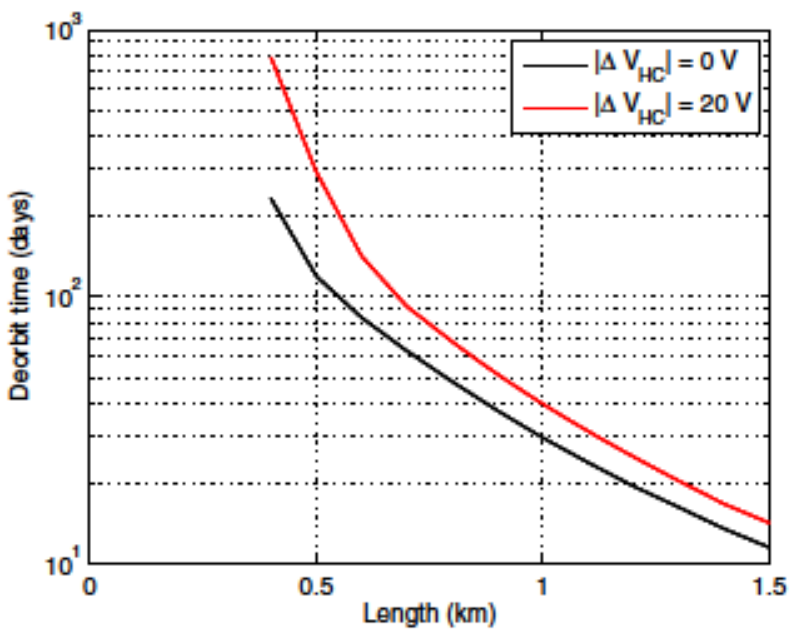

Fig. 6 Deorbit time versus tether length for two $\Delta V_{\mathrm{HC}}$ values. requires an iterative procedure involving models of different complexity [26].

Figure 4, which includes simulations for three different tether widths ( $w^{-}=4,6$, and $8 \mathrm{~cm}$ ), highlights the beyond-OML effect on tether performances. The deorbit times have been computed with $\left(T_{F}\right)$ and without $\left(T_{0}\right)$ the beyond-OML effect, and we found the error: $\left(T_{F}-T_{0}\right) / T_{0}$. The error, which is between 1 and $6 \%$, increases as the tape width is increased and the orbit inclinations are closer to equatorial. We observe that the beyond-OML effect has a limited impact on the deorbit time, and it can be safely neglected for a preliminary tether mission analysis. This result is mainly due to two causes:

1) The slow drop of the ratio $I / I_{\mathrm{OML}}$ as $R / \lambda_{D e}$ is varied [13].

2) The beyond-OML operation mainly occurs at lower altitudes, where the plasma density is high, the Debye length is small, and the tether spends a short time. This issue also explains why the percentage in Fig. 4 is almost constant for inclination above 60 deg.

Figure 5 shows the deorbit time $T_{F}$ versus the initial value of the orbit inclination for different values of the hollow-cathode potential drops $\Delta V_{\mathrm{HC}}$. The simulations were carried by assuming the OML current collection law. Clearly, the $\Delta V_{\mathrm{HC}}$ has an important effect on tether performance for high-inclination orbits and it must be considered in tether mission design. For instance, for an inclination equal to $80 \mathrm{deg}$, one finds $T_{F}=162,223$, and 292 days for $\left|\Delta V_{\mathrm{HC}}\right|=0,20$, and $40 \mathrm{~V}$, respectively.

Very wide and short tethers are seemingly attractive because they are very compact and they present a low probability of severing. To

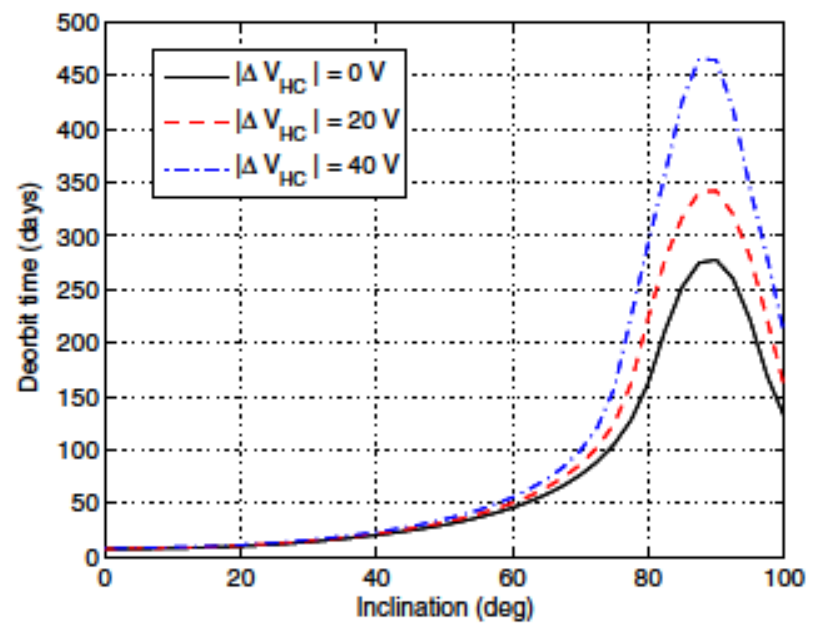

Fig. 5 Deorbit time versus orbit inclination for several $\Delta V_{\mathrm{HC}}$ values. sever the tether requires debris of a size comparable with its transverse dimension, and the debris flux decays fast with the debris size [10]. The deorbit time of a very short tether, however, can be very large, also due to the impact of the hollow-cathode potential drop. This issue is illustrated in Fig. 6, which shows the deorbit time versus the tether length of a $50 \mathrm{~kg}$ satell ite equipped with a tether of a width of $4 \mathrm{~cm}$ and a thickness of $50 \mu \mathrm{m}$. The initial orbit has $60 \mathrm{deg}$ of inclination and $800 \mathrm{~km}$ of altitude, and the simulations are stopped for an altitude equal to $350 \mathrm{~km}$. We observe that the deorbit time increases very rapidly for tethers shorter than about $1 \mathrm{~km}$. The hollow-cathode potential drop effect is also more dramatic as the tether length decreases.

\section{Conclusions}

A high-bias orbital-motion-limited current collection law was used in most past work. As shown in Fig. 3, the high-bias approximation can be safely used. The OML hypothesis, however, is more sensitive and depends on the mission and the tether width. It was remarked that recent work suggests that wide tethers may considerably decrease the probability of severing [10]. As Fig. 4 shows, the difference in tether deorbit times considering the OML and the beyond-OML model are about 1 to $6 \%$. Such an effect can therefore be neglected for preliminary mission analysis but should be included in more advanced simulations.

The beyond-OML effect, as presented here, can be efficiently incorporated into EDT flight simulators. This effect has been included in the characteristic length $L_{*}$, which involves the dimensionless factor $G^{2 / 3} \equiv\left(I / I_{\mathrm{OML}}\right)^{2 / 3}$. Using this new dimensionless variable, the mathematical problem is equal to the classical one $[3,17]$. However, since the factor $G$ may be computed at each time step, its computation by the shooting method explained in [13] can slow down the simulation. This can be solved by interpolation of previously computed values of $G$ or by least-squares fitting. This last procedure together with a semianalytical solution of the tether current and potential profiles will be presented in a forthcoming work [20].

Numerical simulations reveal the impact of the cathodic contactor potential drop $\left|\Delta V_{\mathrm{HC}}\right|$ on the tether performance. This effect, commonly neglected in past work, is particularly relevant for short tethers because the current vanishes if $E_{m} L<\left|\Delta V_{\mathrm{HC}}\right|$. For these missions, one may consider the substitution of the hollow cathode by a fully bare tether with thermionic emission along a cathodic segment of the tether itself, as proposed in [18]. The use of a thin tether coating with a low work function material $(W=0.6 \mathrm{eV}$, emitted current density $1 \mathrm{~A} / \mathrm{m}^{2}$ at $300 \mathrm{~K}$ ) may be an interesting and relatively simple replacement for a hollow cathode. The weight of the hollow-cathode system (about $4-5 \mathrm{~kg}$ ) can be substituted by an increased length of 
tether (for instance, a thermionic segment of $1.5 \mathrm{~km} \times 1.5 \mathrm{~cm} \times$ $100 \mu \mathrm{m}$ ). This would increase the average tether current to a certain factor while avoiding the problem that no current is ejected below certain length ( $E_{m} L>\left|\Delta V_{\mathrm{HC}}\right|$ condition).

\section{Acknowledgment}

This work was carried out under the European Commission's FP7 Bare Electrodynamic Tethers space project (no. 262972).

\section{References}

[1] Sanmartin, J. R., Ahedo, E., and Martinez-Sanchez, M., "An Anodeless Tether Generator," Proceedings of Physics of Charred Bodies in Space Plasmas, edited by Dobrowoiny, M., and Sindotii, E., Editrice Compositori, Bologna, Italy, 1992.

[2] Grossi, M., "Future of Tethers in Space," Proceedings of the Fourth International Conference on Tethers in Space, Science and Technology, U.S. National Aeronautics and Space Administration, Science and Technology Corp., Hampton, VA, 1995, p. 11, http://books.google.es/ books?id=bHCEHAAACAAJ.

[3] Ahedo, E., and Sanmartín, J. R., "Analysis of Bare-Tether Systems for Deorbiting Low-Earth-Orbit Satellites," Journal of Spacecraft and Rockets, Vol. 39, No. 2, March 2002, pp. 198-205. doi: $10.2514 / 2.3820$

[4] Bombardelli, C., Peláez, J., and Sanjurjo, M., "Asymptotic Solution for the Current Profile of Passive Bare Electrodynamic Tethers," Journal of Propulsion and Power, Vol. 26, No. 6, 2010, pp. 1291-1304. doi:10.2514/1.46808

[5] Sanmartin, J. R., Lorenzini, E. C., and Martinez-Sanchez, M., "Electrodynamic Tether Applications and Constraints," Journal of Spacecraft and Rockets, Vol. 47, No. 3, 2010, pp. 442-456. doi: $10.2514 / 1.45352$

[6] Levin, E., Pearson, J., and Carroll, J., "Wholesale Debris Removal from Leo," Acta Astronautica, Vol. 73, April-May 2012, pp. 100-108. doi:10.1016/j.actaastro.2011.11.014

[7] Zhong, R., and Zhu, Z. H., "Libration Dynamics and Stability of Electrodynamic Tethers in Satellite Deorbit," Celestial Mechanics and Dynamical Astronomy, Vol. 116, No. 3, 2013, pp. 279-298. doi:10.1007/s10569-013-9489-4

[8] Zhong, R., and Zhu, Z. H., "Optimal Control of Nanosatellite Fast Deorbit Using Electrodynamic Tether," Journal of Guidance, Control, and Dynamics, Vol. 37, No. 4, 2014, pp. 1182-1194. doi:10.2514/1.G000108

[9] Bombardelli, C., Zanutto, D., and Lorenzini, E., "Deorbiting Performance of Bare Electrodynamic Tethers in Inclined Orbits," Journal of Guidance, Control, and Dynamics, Vol. 36, No. 5, 2013, pp. $1550-1556$. doi: $10.2514 / 1.58428$

[10] Khan, S. B., and Sanmartin, J. R., "Survival Probability of Round and Tape Tethers Against Debris Impact," Journal of Spacecraft and Rockets, Vol. 50, No. 3, May 2013, pp. 603-608. doi:10.2514/1.A32383

[11] Laframboise, J. G., and Parker, L. W., "Probe Design for Orbit-Limited Current Collection," Physics of Fluids, Vol. 16, May 1973, pp. 629-636. doi:10.1063/1.1694398

[12] Sanmartín, J. R., and Estes, R. D., "The Orbital-Motion-Limited Regime of Cylindrical Langmuir Probes," Physics of Plasmas, Vol. 6, No. 1, Jan. 1999, pp. 395-405. doi:10.1063/1.873293

[13] Estes, R. D., and Sanmartín, J. R., "Cylindrical Langmuir Probes Beyond the Orbital-Motion-Limited Regime," Physics of Plasmas,
Vol. 7, No. 10, Oct. 2000, pp. 4320-4325. doi: $10.1063 / 1.1288400$

[14] Choiniere, E., and Gilchrist, G., "Investigation of Ionospheric Plasma Flow Effects on Current Collection to Parallel Wires Using SelfConsistent Steady-State Kinetic Simulations," 41st AIAA/ASME/SAE/ ASEE Joint Propulsion Conference and Exhibit, AIAA Paper 20054293, July 2005

[15] Sanmartín, J. R., "Active Charging Control and Tethers," CNES-Space Technology Course: Prevention of Risks Related to Spacecraft Charging, AIAA, Reston, VA, 2002, pp. 515-533. doi:10.2514/MJPC2005

[16] Sánchez-Arriaga, G., and Pastor-Moreno, D., "Direct Vlasoy Simulations of Electron-Attracting Cylindrical Langmuir Probes in Flowing Plasmas," Physics of Plasmas, Vol. 21, No. 7, 2014, Paper 073504 doi:10.1063/1.4889732

[17] Sanmartin, J. R., Martinez-Sanchez, M., and Ahedo, E., "Bare Wire Anodes for Electrodynamic Tethers," Journal of Propulsion and Power, Vol. 9, No. 3, June 1993, pp. 353-360. doi: $10.2514 / 3.23629$

[18] Chen, X., and Sanmartín, J. R., "Bare-Tether Cathodic Contact Through Thermionic Emission by Low-Work-Function Materials," Physics of Plasmas, Vol. 19, No. 7, July 2012, Paper 073508. doi:10.1063/1.4736987

[19] Sanmartin, J. R., Estes, R. D., and Lorenzini, E. C., Efficiency of Different Types of ED-Tether Thrusters, edited by El-Genk, M. S., American Institute of Physics Conference Series, Vol. 552, American Inst. of Physics, College Park, MD, Feb. 2001, pp. 479-487.

[20] Sanjurjo, M., Sánchez-Arriaga, G., and Peláez, J., "Semi-Analytical Solution of Current Collection in Bare Electrodynamic Tethers," Journal of Aerospace Engineering (submitted for publication).

[21] Sanmartin, J. R., Charro, M., Lorenzini, E. C., Colombatti, G., Roussel, J. F., Sarrailh, P., Williams, J. D., Xie, K., García de Quirós, F., Carrasco, J. A., Rosta, R., Zoest, T., Lasa, J., and Marcos, J., "BETs: Propellant Less Deorbiting of Space Debris by Bare Electrodynamic Tethers," Let's Embrace Space, European Commission, Brussels, 2012, pp. 506-513. doi: $10.2769 / 31208$

[22] Pelaez, J., Hedo, J. M., and de Andres, P. R., "A Special Perturbation Method in Orbital Dynamics," Celestial Mechanics and Dynamical Astronomy, Vol. 97, No. 2, 2007, pp. 131-150. doi:10.1007/s10569-006-9056-3

[23] Bau, G., Bombardelli, C., and Peláez, J., "A New Set of Integrals of Motion to Propagate the Perturbed Two-Body Problem," Celestial Mechanics and Dynamical Astronomy, Vol. 116, No. 1, 2013, pp. 53-78 doi:10.1007/s10569-013-9475-x

[24] Bau, G., and Bombardelli, C., "Time Elements for Enhanced Performance of the DROMO Orbit Propagator," Astronomical Journal, Vol. 148, No. 3, 2014, p. 43. doi:10.1088/0004-6256/148/3/43

[25] Liou, J. C., and Johnson, N. L., "A Sensitivity Study of the Effectiveness of Active Debris Removal in LEO," Acta Astronautica, Vol. 64, Nos. 2-3, 2009, pp. 236-243. doi:10.1016/i.actaastro.2008.07.009

[26] Mantellato, R., Valmorbida, A., Pertile, M., Francesconi, F., Lorenzini, E. C., and Sánchez-Arriaga, G., "End-of-Life Deorbiting Services for Small Satellites Making Use of Bare Electrodynamic Tethers," 4th Small Satellites and Services Symposium, ESA-ESTEC, Noordwijk, The Netherlands, May 2014

A. Gallimore Associate Editor 